$\mathrm{PSU} / \mathrm{TH} / 184$

June 1997

\title{
Closing the window on the axigluon mass using top quark production data
}

\author{
M. A. Doncheski \\ Department of Physics \\ The Pennsylvania State University \\ Mont Alto, PA 17237 USA \\ and \\ R. W. Robinett \\ Department of Physics \\ The Pennsylvania State University \\ University Park, PA 16802 USA
}

\begin{abstract}
The contribution of axigluons (the massive color-octet gauge bosons in all chiral color models) to top quark pair production in hadronic collisions is considered. The agreement between the experimental values of the $t \bar{t}$ production cross-section at the TEVATRON and recent QCD predictions is used to discuss limits on the axigluon mass. Specifically, intermediate mass axigluons, those in the mass range $50 \mathrm{GeV}<M_{A}<120 \mathrm{GeV}$ which has not already been excluded, would increase the tree-level $q \bar{q} \rightarrow t \bar{t}$ cross-section by a factor of $\geq 2$, thereby increasing the theoretical predictions for $\sigma_{t \bar{t}}$ by $\Delta \sigma_{t \bar{t}}=3.2-3.7 p b(2.7-3.1 p b)$ using leading-order (next-to-leading order) parton distributions over this mass range, independent of the axigluon decay width. Such an increase is roughly $1.3-1.6(0.9-1.2)$ standard deviations larger than that suggested by the apparent good agreement between combined experimental results and recent theoretical calculations and so is not ruled out, but is definitely disfavored. Future high-statistics top-quark production runs will likely make a more definitive statement. The forward-backward asymmetry in $t \bar{t}$ production induced by axigluons in this mass window is also discussed and found to be quite large and so could provide another constraint.
\end{abstract}


Extensions of the standard model of QCD which enlarge the color group to $S U_{3}(L) \times$ $S U_{3}(R)$ at high energies, so-called chiral color models [1], all predict the existence of a massive, color-octet gauge boson which couples to quarks with an axial vector coupling and the same strong interaction strength as QCD. Such a particle would make dramatic changes in heavy quarkonium decays and limits from $\Upsilon$ decays involving real [2], [3] or virtual [四] axigluons have been used to restrict their mass to $M_{A} \geq 25 \mathrm{GeV}$ where $M_{A}$ is the axigluon mass. A more stringent lower bound on $M_{A}$ can be derived by consideration of its possible contribution to the $R$ ratio in $e^{+} e^{-}$collisions with a firm bound of $M_{A} \geq 50 \mathrm{GeV}$ now accepted [5]. Bagger, Schmidt, and King [6] noted that axigluon production in hadronic collisions could give rise to observable resonance structures in the di-jet cross-section and various groups have found the following overlapping limits on axigluon masses (usually with some assumptions made about the axigluon decay width)

$$
\begin{array}{lll}
\text { UA1 } & \text { Ref. [7] } & 150<M_{A}<310 \mathrm{GeV} \\
\text { CDF } & \text { Ref. [1] } & 120<M_{A}<210 \mathrm{GeV} \\
\text { CDF } & \text { Ref. [9] } & 200<M_{A}<870 \mathrm{GeV} \\
\text { CDF } & \text { Ref. [10 } & 200<M_{A}<980 \mathrm{GeV}
\end{array}
$$

We combine these limits in Fig. 1 as an excluded band $\left(120<M_{A}<980 \mathrm{GeV}\right)$ along with the lower bound from $e^{+} e^{-}$interactions. These bounds are the most restrictive ones quoted in the most recent Review of Particle Properties [11] and indicate that there is still an allowed window for intermediate mass axigluons in the range $50 \mathrm{GeV}<$ $M_{A}<120 \mathrm{GeV}$.

Axigluons can also contribute to heavy flavor production by their contribution to the sub-process $q \bar{q} \rightarrow g, A \rightarrow Q \bar{Q}$ and in this note we consider what limits, if any, top quark production data can set on intermediate mass (or very heavy) axigluons. The 
tree-level cross-section for top-quark production from a $q \bar{q}$ initial state including both $s$-channel gluons and axigluons is given by [12]

$$
\left(\frac{d \sigma}{d \hat{t}}\right)_{q \bar{q}}=\left(\frac{d \sigma}{d \hat{t}}\right)_{0}\left[1+|r(\hat{s})|^{2}+4 \operatorname{Re}(r(\hat{s})) \frac{(\hat{t}-\hat{u}) \hat{s} \beta}{(\hat{t}-\hat{u})^{2}+\hat{s}^{2} \beta^{2}}\right]
$$

where $\beta=\sqrt{1-4 M_{t}^{2} / \hat{s}}$ and $M_{t}$ is the top quark mass. The standard gluon-induced result is given by

$$
\left(\frac{d \sigma}{d \hat{t}}\right)_{0}=\frac{1}{16 \pi \hat{s}^{2}} \frac{64 \pi^{2}}{9} \alpha_{s}^{2}\left[\frac{\left(M_{t}^{2}-\hat{t}\right)^{2}+\left(M_{t}^{2}-\hat{u}\right)^{2}+2 M_{t}^{2} \hat{s}}{\hat{s}^{2}}\right]
$$

and the enhancement factors due to axigluons depend on the ratio

$$
r(\hat{s})=\frac{\hat{s}}{\hat{s}-M_{A}^{2}+i M_{A} \Gamma_{A}}
$$

where $\Gamma_{A}$ is the axigluon decay width. We also have assumed that the axigluonquark and gluon-quark couplings both have strength $g_{s}$. The last term in square brackets in Eqn. (11) is due to the interference between the gluon- and axigluon-induced processes and gives no contribution in the total cross-section when integrated over parton distributions and phase space as it is odd in $\cos (\Theta)$ where $\Theta$ is the centerof-mass angle. It does, however, contribute to a forward-backward asymmetry in $t \bar{t}$ production [12 which we discuss below. Any increase in the top-quark production cross-section is then due to the second term, $|r(\hat{s})|^{2}$, in Eqn. (11).

In order to compare to existing top-quark production data from the TEVATRON, we evaluate the increase in the $t \bar{t}$ production cross-section due to this term for various values of $M_{A}$; we use fixed values of $M_{t}=175 \mathrm{GeV}$ and $\sqrt{s}=1.8 \mathrm{TeV}$. Because top quark production is dominated by the $q \bar{q}$ fusion mechanism at Born level, changes in this process can have a more obvious and direct effect on heavy quark production 
in this case than say for $b$-quark production where $g g \rightarrow b \bar{b}$ dominates. In fact, at the TEVATRON where the average center-of-mass energy for $t \bar{t}$ production is much larger than $M_{A}$ in the intermediate mass regime (namely $\langle\sqrt{\hat{s}}\rangle \approx 450 \mathrm{GeV}>2 M_{t} \approx$ $350 \mathrm{GeV}>>(50-120 \mathrm{GeV})$, the effect of axigluons in this mass range would be to more than double the already dominant tree-level $q \bar{q}$ production cross-section for top quark production since $|r(\hat{s})| \geq 1$ in this region.

The increase in the $t \bar{t}$ cross-section due to axigluons is plotted in Fig. 1 as a function of $M_{A}$ for several different sets of assumptions; we normally assume an axigluon decay width given by $\Gamma_{A}=N \alpha_{s} M_{A} / 6 \approx 0.1 M_{A}$ assuming $N=5$ light flavors of quarks as the main decay mode. The solid curve makes use of a set of parton distributions which is extracted from data using a leading-order (LO) analysis [13 as might be considered most appropriate for this leading order calculation. The dashed curve makes use of a recent, next-to-leading (NLO) set (MRRS, mode=1, $m_{c}=1.35 \mathrm{GeV}$, 114) and gives somewhat lower values; another popular NLO parameterization (CTEQ4M, 15]) gives almost identical results to this one. The dot-dash curve uses the LO parton distributions, but assumes an axigluon width of $\Gamma_{A}=0.2 M_{A}$; increasing $\Gamma_{A}$ has a negligible effect for small axigluon masses, but decreases the cross-section somewhat for larger values of $M_{A}$. As mentioned above, for very low mass axigluons $\left(M_{A}<<2 M_{t}\right)$, the cross-section is essentially doubled from its $q \bar{q}$-induced tree-level value and it is this region on which we will focus our attention. (The unacceptably huge resonant production contribution for $M_{A} \approx 2 M_{t}$ would easily eliminate the region $200 \mathrm{GeV}<$ $M_{A}<780 \mathrm{GeV}$ which is already excluded by di-jet analyses.) In the intermediate-mass range which is not already excluded, the tree-level $q \bar{q}$-induced cross-section is more than doubled and $\sigma_{t \bar{t}}$ would increase by $3.3-3.7 p b(2.7-3.1 p b)$ using LO (NLO) parton distributions if axigluons in this mass range were present. 
Starting with the first observation of the top quark at the TEVATRON [16], [17], there has been an increasingly large set of data on the $t \bar{t}$ production cross-section in a variety of channels. The experimental situation has been recently reviewed by Gerdes [18] who cites combined results (for dilepton and $l+$ jets channels) for the D0 and CDF groups as

$$
\begin{array}{ll}
\sigma_{t \bar{t}}\left(M_{t}=173.3 \mathrm{GeV}\right)=5.5 \pm 1.8 \mathrm{pb} & \mathrm{D} 0 \\
\sigma_{t \bar{t}}\left(M_{t}=175.0 \mathrm{GeV}\right)=7.5_{-1.6}^{+1.9} \mathrm{pb} & \mathrm{CDF}
\end{array}
$$

Increasingly more sophisticated QCD calculations of the top-quark production crosssection, including resummation effects, have become available and two recent groups find

$$
\begin{array}{lll}
\text { Berger and Contopanagos } & \text { Ref. } 19] & \sigma_{t \bar{t}}\left(M_{t}=175 \mathrm{GeV}\right)=5.52_{-0.42}^{+0.07} p b \\
\text { Catani et al } & \text { Ref. }[20] & \sigma_{t \bar{t}}\left(M_{t}=175 \mathrm{GeV}\right)=4.75_{-0.62}^{+0.73} p b
\end{array}
$$

Given the relatively large statistical errors on the experimental measurements, the theoretical and experimental values agree reasonably well and arguably leave little room for a large new top-quark production mechanism. While we will not perform a detailed statistical analysis, we can make some simple comments. If we average the experimental values and theoretical predictions (combining their errors) and ask for the difference between experiment and theory, we find very roughly that

$$
\overline{\Delta \sigma}=\overline{\sigma_{t \bar{t}}(\exp )}-\overline{\sigma_{t \bar{t}}(t h)} \approx 1.4 \pm 1.4 p b
$$

which is certainly consistent with zero. This implies that an additional $\Delta \sigma_{t \bar{t}}=3.2-$ $3.7 p b(2.4-3.1 p b)$ contribution from axigluon intermediate states is disfavored, but only at the level of something like $1.3-1.6 \sigma(0.9-1.2 \sigma)$ using LO (NLO) parton distributions in this leading-order analysis. The 50-fold increase in statistics expected for $t \bar{t}$ production during the next TEVATRON run (due to a higher $\sqrt{s}=2 \mathrm{TeV}$ and 
more luminosity) will dramatically reduce the statistical errors quoted above and allow for a much better comparison with both standard QCD and extensions thereof. If the complete set of NLO predictions for the combined gluon plus axigluon processes gave rise to a similarly large increase in cross-section as has been observed for the pure QCD case (something like a $44 \%$ increase over LO), the disagreement with data would increase even further.

It was noted some time ago [12] that axigluon intermediate states could give rise to a large forward-backward asymmetry in heavy quark production, due to the interference term in Eqn. (11). If we define $y=\cos (\Theta)$ as the center-of-mass angle for the $2 \rightarrow 2$ $q \bar{q} \rightarrow Q \bar{Q}$ process, then the forward-backward asymmetry (at Born level) can be defined as

$$
A_{F B}=\frac{\sigma_{q \bar{q}}(y>0)-\sigma_{q \bar{q}}(y<0)}{\sigma_{q \bar{q}}(y>0)+\sigma_{q \bar{q}}(y<0)+\sigma_{g g}}
$$

where Born-level $g g$ process is evaluated using standard expressions [21]. Once again, for fixed values of $M_{t}=175 \mathrm{GeV}, \sqrt{s}=1.8 \mathrm{TeV}$, LO parton distributions, and $\Gamma_{A}=$ $0.1 M_{A}$ we plot this asymmetry in Fig. 2 versus $M_{A}$. The forward-backward asymmetry in the allowed intermediate mass region of interest is very large $(\sim 75 \%)$ and does not vary much with parton distributions or axigluon width. More detailed tests of the top quark production process might well be used to probe this effect and further constrain axigluon masses.

\section{Acknowledgments}

One of us (M.A.D) acknowledge the support of Penn State University through a Research Development Grant (RDG). 


\section{References}

[1] P. H. Frampton and S. L. Glashow, Phys. Lett. 190B, (1987) 157; Phys. Rev. Lett. 58, (1987) 2168.

[2] F. Cuypers and P. H. Frampton, Phys. Rev. Lett. 60, (1988) 1237.

[3] M. A. Doncheski, H. Grotch, and R. Robinett, Phys. Lett. 206B, (1988) 137.

[4] M. A. Doncheski, H. Grotch, and R. W. Robinett, Phys. Rev. D38, (1988) 412.

[5] F. Cuypers, A. F. Falk, and P. H. Frampton, Phys. Lett. 259, (1991) 173.

[6] J. Bagger, C. Schmidt, and S. King, Phys. Rev. D37, (1988) 1188.

[7] C. Albajar et al. (UA1 Collaboration), Phys. Lett. 209B, (1988) 127.

[8] F. Abe et al. (CDF Collaboration), Phys. Rev. D41, (1990) 1722.

[9] F. Abe et al. (CDF Collaboration), Phys. Rev. Lett. 74, (1995) 3538.

[10] F. Abe et al. (CDF Collaboration) Phys. Rev. D55, (1997) R5263.

[11] Review of Particle Physics, Particle Data Group, Phys. Rev. 54, (1996) 1; see page 236 for the detailed review.

[12] L. M. Sehgal and M. Wanninger, Phys. Lett. 202B, (1988) 211.

[13] J. F. Owens, Phys. Lett. B266, (1991) 126.

[14] A. D. Martin, R. G. Roberts, M. G. Ryskin, and W. J. Stirling, Durham preprint DTP-96-102, hep-ph/9612449. 
[15] H.L. Lai, J. Huston, S. Kuhlmann, F. Olness, J. Owens, D. Soper, W.K. Tung, and H. Weerts, Phys. Rev. D55, (1997), 1280.

[16] F. Abe et al, Phys. Rev. Lett. 74, (1995) 2626.

[17] S. Abachi et al., Phys. Rev. Lett. 74, (1995) 2632.

[18] D. W. Gerdes (for the CDF collaboration), FERMILAB-CONF-97/166-E, hepex/9706001, to appear in the proceedings of the XXXIInd Rencontres de Moriond, Electroweak Interactions and Unified Theories, Les Arcs, Savoie, France, March 1997.

[19] E. L. Berger and H. Contopanagos, Phys. Rev. D54, (1996) 3085; Argonne report ANL-HEP-CP-97-33, hep-ph/9706356. to appear in the Proceedings of DIS97, Fifth International Workshop on Deep Inelastic Scattering and QCD, Chicago, IL, April 1997.

[20] S. Catani, M. L. Mangano, P. Nason, and L. Trentadue, Phys. Lett. B378, (1996) 329.

[21] B. Combridge, Nucl. Phys. B151, (1979) 429. 


\section{Figure Captions}

Fig. 1. Additional contribution to the $t \bar{t}$ production cross-section due to axigluons (in $p b$ ) for $p \bar{p}$ collisions at $\sqrt{s}=1.8 \mathrm{TeV}$ using $M_{t}=175 \mathrm{GeV}$ versus mass of the axigluon $\left(M_{A}\right)$ in $\mathrm{GeV}$. The curves correspond to LO parton distributions [13] and $\Gamma_{A}=0.1 M_{A}$ (solid), NLO partons [14] and $\Gamma_{A}=0.1 M_{A}$ (dashed), and LO partons [13] and $\Gamma_{A}=0.2 M_{A}$ (dot-dash). Excluded regions for the axigluon mass from collider dijet data and $e^{+} e^{-}$analyses are also shown.

Fig. 2. Forward-backward asymmetry $A_{F B}$, defined in Eqn. (5), for $t \bar{t}$ production in $p \bar{p}$ collisions (assuming $M_{t}=175 \mathrm{GeV}$ and $\sqrt{s}=1.8 \mathrm{TeV}$ ) versus axigluon mass using LO partons 13 and $\Gamma_{A}=0.1 M_{A}$. Also shown are published limits on the axigluon mass. 


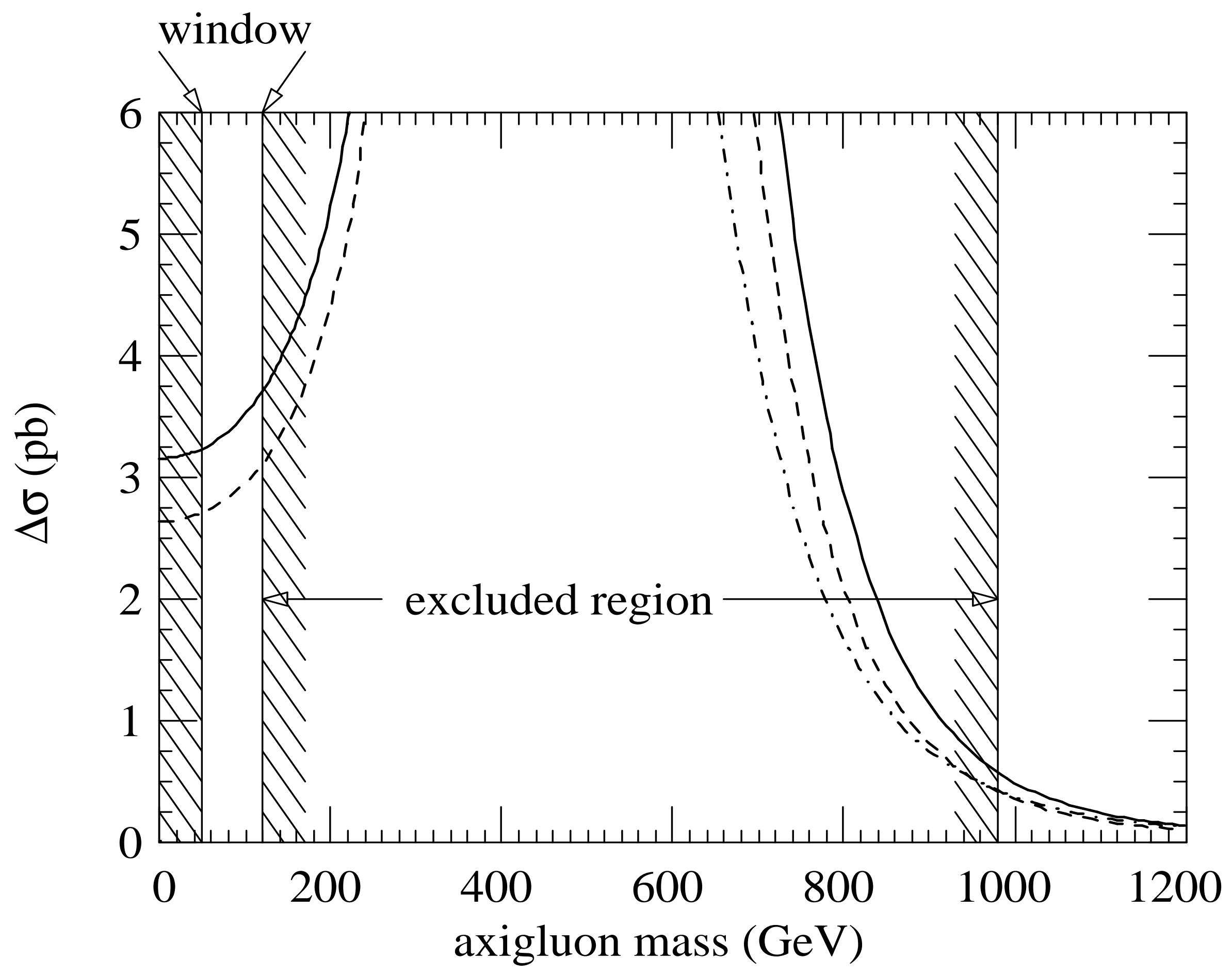




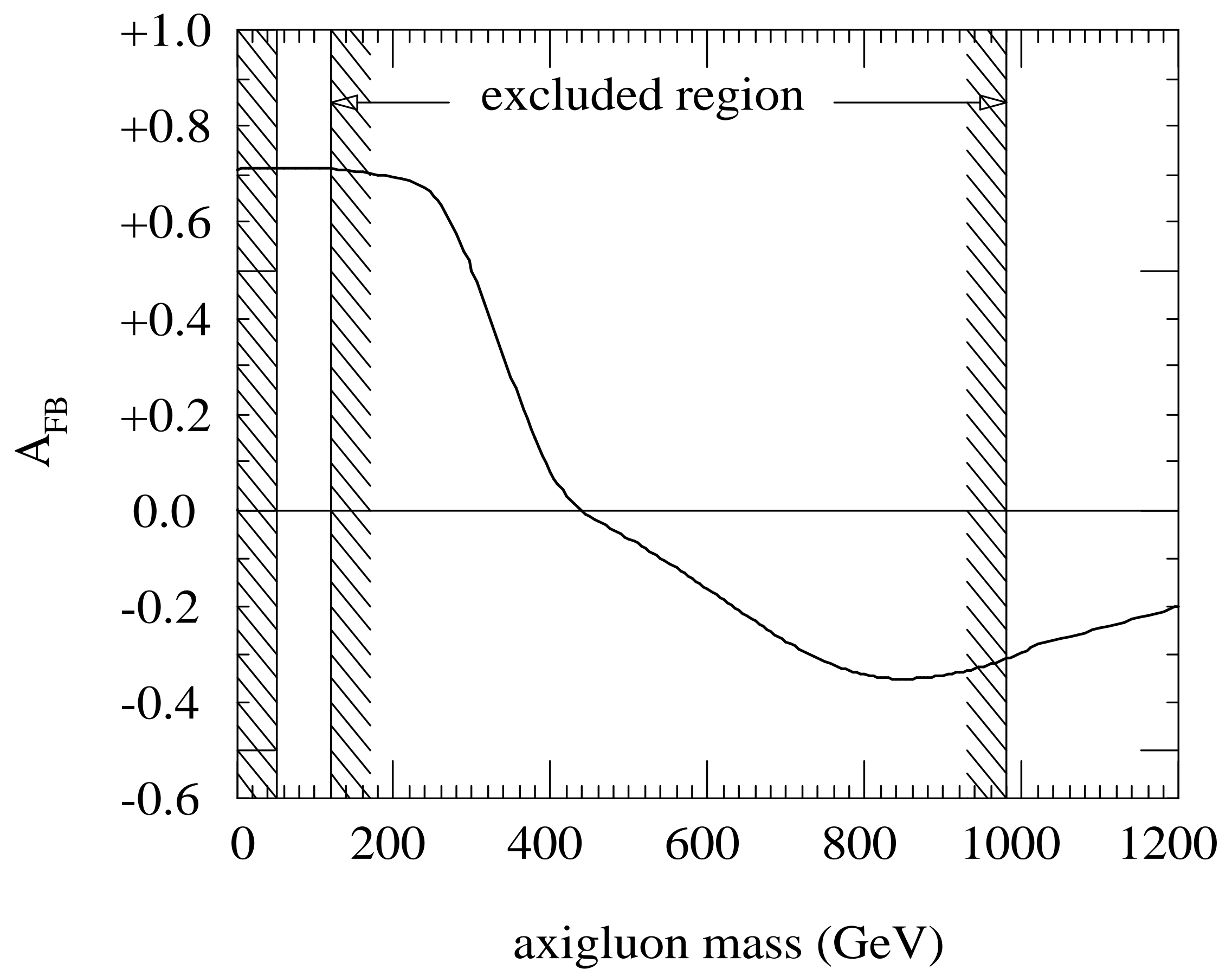

